\title{
Modulational stability and dark solitons in periodic quadratic nonlinear media
}

\section{Corney, Joel Frederick; Bang, Ole}

Published in:

Nonlinear Optics: Materials, Fundamentals, and Applications, 2000. Technical Digest

Link to article, DOI:

10.1109/NLO.2000.883676

Publication date:

2000

Document Version

Publisher's PDF, also known as Version of record

Link back to DTU Orbit

Citation (APA):

Corney, J. F., \& Bang, O. (2000). Modulational stability and dark solitons in periodic quadratic nonlinear media. In Nonlinear Optics: Materials, Fundamentals, and Applications, 2000. Technical Digest (pp. 362-364). https://doi.org/10.1109/NLO.2000.883676

\section{General rights}

Copyright and moral rights for the publications made accessible in the public portal are retained by the authors and/or other copyright owners and it is a condition of accessing publications that users recognise and abide by the legal requirements associated with these rights.

- Users may download and print one copy of any publication from the public portal for the purpose of private study or research.

- You may not further distribute the material or use it for any profit-making activity or commercial gain

- You may freely distribute the URL identifying the publication in the public portal 


\title{
Modulational stability and dark solitons in periodic quadratic nonlinear media
}

\author{
J. F. Corney and O. Bang \\ Department of Mathematical Modelling, Technical University of Denmark, \\ DK-2800 Lyngby, Denmark \\ Ph: +45 45253108, Fax: +45 45931235,jfc@imm.dtu.dk
}

\begin{abstract}
We show that stable dark solitons exist in quadratic nonlinear media with periodic linear and nonlinear susceptibilities. We investigate the modulational stability of plane waves in such systems, a necessary condition for stable dark solitons.

(C) 2000 Optical Society of America

OCIS codes: (190.5530) Pulse propagation and solitons; (230.4320) Nonlinear optical devices; (190.4410) Non-

linear optics, parametric processes; (190.5940) Self-action effects
\end{abstract}

Optical materials with $\chi^{(2)}$ nonlinearity offer many advantages as candidates for all-optical processing applications. For example, through cascading $\chi^{(2)}: \chi^{(2)}$ processes, they offer a fast and strong nonlinear response, and they can support localised solitary-wave solutions in more than one dimension, in contrast with systems that have only a cubic nonlinear response. However materials with only quadratic nonlinearity are unable to support stable dark solitons, which have potential application as steerable, self-trapping wave-guides. In useful $\chi^{(2)}$ materials, the intrinsic cubic nonlinearity is usually too weak to stabilise dark solitons against modulational instability[1].

Here we consider simultaneous linear and nonlinear gratings in a $\chi^{(2)}$ medium, which can induce cubic nonlinearities $[2,3]$ and partially reduce the effective quadratic nonlinearity $[4,5]$. We investigate the possibility of having induced cubic terms that are large enough to stabilise dark solitons against modulational instability in a system that still has a strong effective quadratic response. Even though the induced cubic nonlinearity may be stronger than the intrinsic nonlinearity and is of a fundamentally different nature, we find that it is insufficient to ensure stability, without at least a partial reduction in the effective quadratic nonlinearity.

We begin with normalised time-independent envelope equations for the fundamental $w$ and second harmonic $v$ components of the electric field $[6,7]$ :

$$
0=\left(i \frac{\partial}{\partial z}+\frac{1}{2} \frac{\partial^{2}}{\partial x}+\Xi^{(1)}(z)\right) w+\chi(z) w^{*} v e^{i \Delta k z} ; \quad 0=\left(i \frac{\partial}{\partial z}+\frac{1}{4} \frac{\partial^{2}}{\partial x}+2 \Xi^{(2)}(z)\right) v+\chi(z) w^{2} e^{-i \Delta k z},
$$

where $\Xi^{(j)}(z)$ is the normalised linear susceptibility at the fundamental $(j=1)$ and second harmonic $(j=2)$ frequencies, and $\chi(z)$ is the nonlinear grating. For wave numbers $k_{1}$ and $k_{2}$ of the fundamental and second harmonic waves, we define the wave-number mismatch as $\Delta k=\left(k_{2}-2 k_{1}\right)$, which is positive for normal dispersion and negative for anomalous dispersion. We consider gratings for forward-wave first-order quasiphase matching, so that the spatial frequency must be $K \simeq \Delta k$. This means that the grating period will be much larger than the optical period, thereby minimizing any Bragg reflection effects.

The case without the linear grating has been dealt with elsewhere[2]. We use the same technique to derive approximate equations for the average fields $w_{0}$ and $v_{0}$ in the more general situation considered here. We assume that the residual phase mismatch is small $(\beta=\Delta k-K \ll K)$ and that the Fourier components of the fields change little over a grating period. The resulting expressions take into account the lowest-order perturbations, proportional to the grating wavelength $L=2 \pi /|K|$. They are

$0=\left(i \frac{\partial}{\partial z}+\frac{1}{2} \frac{\partial^{2}}{\partial x}\right) w_{0}+\rho w_{0}^{*} v_{0}+\gamma\left(\left|v_{0}\right|^{2}-\left|w_{0}\right|^{2}\right) w_{0} ; \quad 0=\left(i \frac{\partial}{\partial z}+\frac{1}{4} \frac{\partial^{2}}{\partial x}+\beta\right) v_{0}+\rho^{*} w_{0}^{2}+2 \gamma\left|w_{0}\right|^{2} v_{0}$

where

$$
\rho=\frac{2 i}{\pi}\left(d+\frac{2 d_{0}}{K}\left(\kappa^{(1)}-\kappa^{(2)}\right)\right) ; \quad \gamma=\frac{1}{K}\left(d_{0}^{2}+d^{2}\left(1-\frac{8}{\pi^{2}}\right)\right) .
$$

Here we have assumed a square grating, in which $d_{0}$ is the DC component of the nonlinear grating and $2 d$ is the modulation depth. The modulation depths of the linear grating are $2 \kappa^{(1)}$ and $2 \kappa^{(2)}$, as illustrated in Figure 1. 

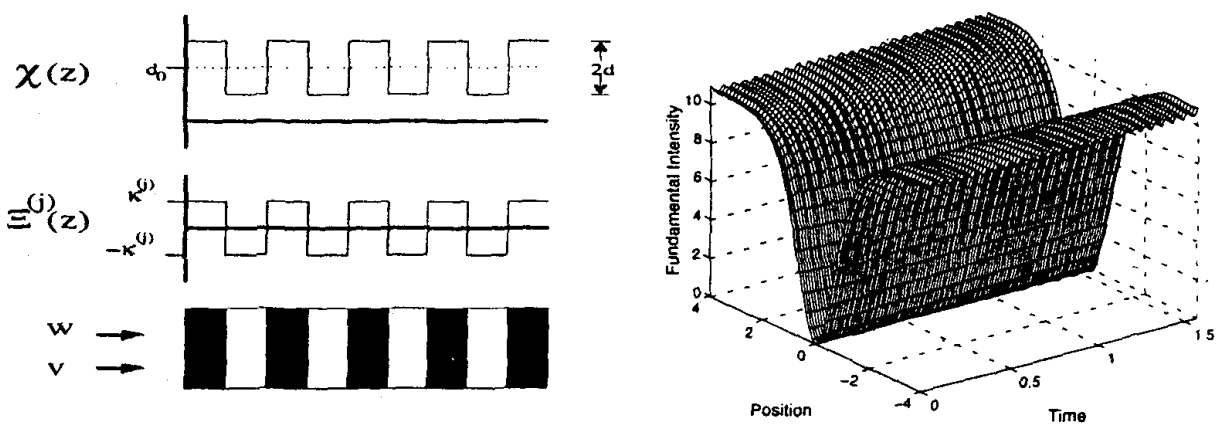

Fig. 1. LEFT: Square-wave modulation, $\Xi^{(j)}(z)$ and $\chi(z)$, of the linear and nonlinear susceptibilities, respectively. RIGHT: Cubic (tanh) dark soliton propagation in the dual grating, showing the evolution of the fundamental intensity. Here $K=100$ and $\beta=0$, with soliton parameter $\lambda=1$ and initial normalised transverse wave number $\Omega=0.049$.

Dark soliton solutions to these first-order equations have not been considered before, partly because the $\gamma>0$ branch has not been investigated. We consider bright soliton for both signs of $\gamma$ in another contribution. A necessary condition for the existence of stable dark solitons is the modulational stability of the background plane wave. In a system with only quadratic nonlinearity, plane waves are modulationally unstable; there must be some appropriate cubic nonlinearity to generate regions of stability[1]. We see in the first-order equations that the nonlinear grating has induced cubic nonlinearities, as well as a residual quadratic nonlinearity. The size of these effective nonlinearities depends on the grating parameters: the quadratic coefficient $\rho$ depends on both the linear and nonlinear gratings, and could in some situations be very small, or even negligible $[4,5]$. The cubic nonlinearity may thus be large enough compared to the quadratic term, and of the correct sign, to enable modulational stability. Dual-period nonlinear gratings also produce similar results[3].

We consider first the case in which the effective quadratic nonlinearity is cancelled $(\rho=0)$, which can occur for realistic physical parameters. In the absence of the parametric coupling term, nontrivial solutions exist even when the second harmonic field contains a vacuum $\left(v_{0}=0\right)$, in which case the first-order equations reduce to the well known nonlinear Schrödinger (NLS) equation, which is known to have stable dark soliton solutions when $\gamma>0$. Figure 1 shows a simulation of the original field equations [Eq. (I)] when the fundamental field has been excited with a tanh dark soliton. No transient shedding of radiation occurs, and the fundamental intensity contains only rapid, small oscillations superimposed on the large average beam. If, as in previous work $[4,5]$, the induced cubic nonlinearities are ignored, then no solitons are predicted to exist.

Even though this situation with $\rho=0$ permits stable dark enlitons, it is at the expense of the fast, strong quadratic nonlinearity. Hence we now consider the case when some residual quadratic nonlinearity remains (i.e. $\rho \neq 0$ ), and apply the standard techniques of modulational stability analysis $[1,8]$ to plane-wave solutions of Eq. (2). To consider plane waves with longitudinal wave-number offset $\Lambda$ and transverse wave-number $\Omega$, we transform to the new field variables $w_{0}=\sqrt{\eta} w^{\prime} e^{i \Lambda z-i \Omega x} /|\rho|, v_{0}=\sqrt{\eta} v^{\prime} e^{2 i \Lambda z-2 i \Omega x} / \rho$, where $\eta=\left|\Lambda+\frac{1}{2} \Omega^{2}\right|$. Both $w^{\prime}$ and $v^{\prime}$ are real, and we take $w^{\prime}$ to be positive, through a gauge transformation. The resultant equations are simplified by a Galilean transformation to a frame that moves at the phase velocity of the plane wave: $x^{\prime}=\sqrt{\eta}(x+\Omega z)$ and $z^{\prime}=\eta z$. They are:

$$
0=\left(i \partial_{z^{\prime}}+\frac{1}{2} \partial_{x^{\prime}}^{2}\right) w^{\prime}+w^{\prime} v^{\prime}+\alpha\left(v^{2}-w^{2}\right) w^{\prime}-r w^{\prime} ; \quad 0=\left(i \partial_{z^{\prime}}+\frac{1}{4} \partial_{x^{\prime}}^{2}\right) v^{\prime}+w^{\prime 2}+2 \alpha w^{\prime 2} v^{\prime}-\sigma v^{\prime},
$$

where $\alpha=\sqrt{\eta} \gamma /|\rho|, r=\operatorname{sgn}\left(\Lambda+\frac{1}{2} \Omega^{2}\right)$ and $\sigma=2 r-\beta / \eta$. Any plane wave will correspond to a constant solution of Eq. (4). For a given $r$, there are up to three branches of solutions for $w^{\prime}$ and $v^{\prime}$, which are depicted in Fig. 2 for $r=-1$. Note that the $r=-1$ solutions can be transformed to the $r=1$ solutions by replacing $v^{\prime} \rightarrow-v^{\prime}, \sigma \rightarrow-\sigma$ and $\alpha \rightarrow-\alpha$. However the modulational stability properties differ. Not shown are the trivial solution $w^{\prime}=v^{\prime}=0$ and the degenerate solution $w^{\prime}=0, v^{\prime}$ arbitrary that exists for $\sigma=0$. Even though this degenerate solution does possess regions of modulational stability, it does not correspond to any dark soliton solution, since there is no nonlinearity in the equation for $v^{\prime}$ when $w^{\prime}=0$. 

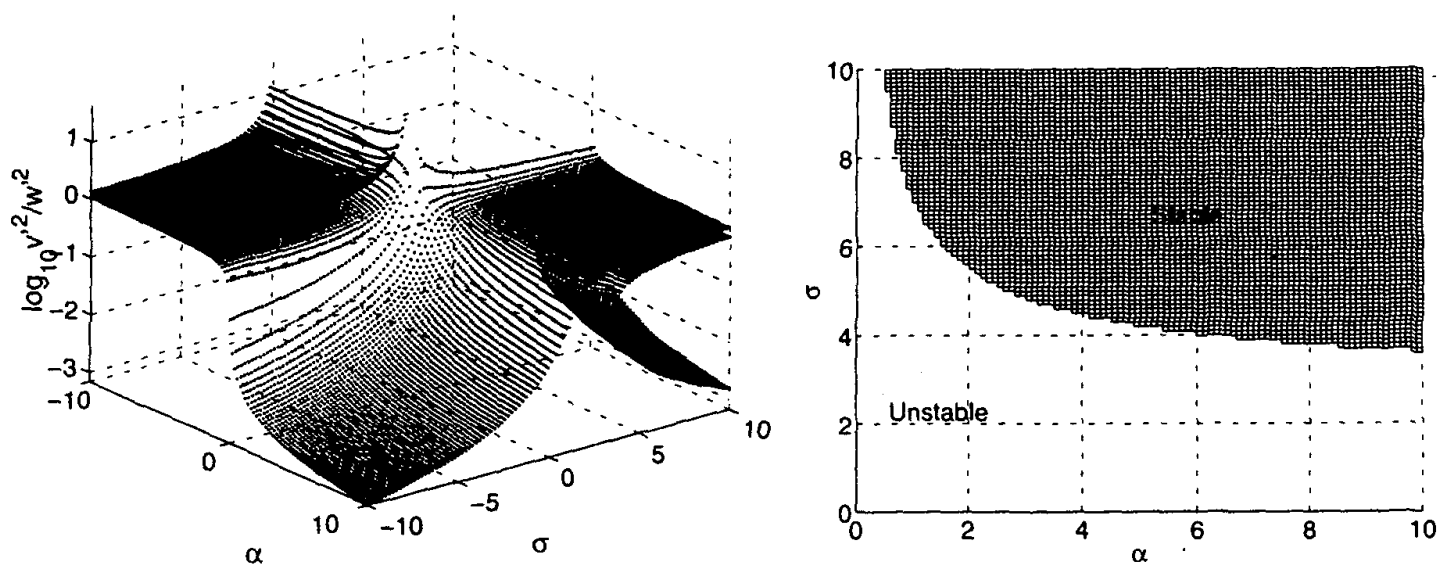

Fig. 2. LEFT: Ratio of intensities for plane-wave solutions, as a function of $\sigma$ and $\alpha$, for $r=-1$. RIGHT: Region of stability for the $r=-1$ solutions.

By using these solutions, the growth of perturbations of the form $\Delta w^{\prime(j)}=\Delta w_{1}^{\prime(j)} e^{\Gamma z^{\prime}-i \nu x^{\prime}}+\Delta w_{2}^{\prime(j)^{*}} e^{\Gamma z^{\prime}+i \nu x^{\prime}}$ is analysed, where * denotes the complex conjugate. A positive gain $\Gamma$ for any frequency $\nu$ indicates modulational instability. For $r=1$ and $r=0$, stability analysis shows that all non-degenerate and non-trivial solutions are unstable.

For the $r=-1$ solutions, however, one of the branches that exists for $\alpha, \sigma>0$ is stable. Figure 2 shows the region of stability, which differs significantly from that found in [1], since there is no self-phase nonlinearity for $v_{0}$ in Eq. (2). In the limit as $\alpha \rightarrow \infty$, we recover the stable NLS solution. This figure reveals that stable waves exist even for a finite $\alpha$, but that $\alpha$ and $\sigma$ cannot both be small. This means that the size of the effective quadratic nonlinearity $\rho$ may be limited. It is true that $\rho$ can be increased at the expense of increasing $-\beta$. However $|\beta|$ is limited by the assumption $|\beta| \ll|K|$ that was used in deriving Eq. (2). Other methods, which go beyond the first-order equations, are being investigated.

To confirm these stability results, and to determine the limits of the validity of the first-order equations, we perform numerical simulations of the field equations, with plane waves seeded with Gaussian noise as the initial conditions. Preliminary results appear to confirm that the effective quadratic nonlinearity must be reduced (by the linear grating) to achieve modulational stability.

\section{References}

1. T. J. Alexander, A. V. Buryak and Y. S. Kivshar, "Stabilization of dark and vortex parametric spatial solitons," Opt. Lett. 23, 670-672 (1998)

2. C. B. Clausen, O. Bang and Y. S. Kivshar, "Spatial solitons and induced Kerr effects in quasi-phase-matched quadratic media," Phys. Rev. Lett. 78, 4749-4752 (1997).

3. O. Bang, C. B. Clausen, P. L. Christiansen and L. Torner, "Engineering competing nonlinearities," Opt. Lett. 24, 1413$1415(1999)$

4. M. M. Fejer, G. A. Magel, D. H. Jundt, and R. L. Byer, "Quasi-phase-matched second harmonic generation: Tuning and tolerances," IEEE Journal of Quantum Electronics 28, 2631-2654 (1992).

5. T. Suhara and H. Nishihara, "Theoretical analysis of waveguide second-harmonic generation phase matched with uniform and chirped gratings," IEEE Journal of Quantum Electronics 26, 1265-1276 (1990); B. Jaskorzynska, G. Arvidsson, and F. Laurell, "Periodic structures for phase- matching in second harmonic generation in titanium lithium niobate waveguides," in Spie: Integrated Optical Circuit Engineering III 251, 221-228 (1986); D. V. Petrov, "Nonlinear phase shift by cascaded quasi-phase-matched second harmonic generation," Opt. Commun. 131, 102-106 (1996).

6. J. A. Armstrong, N. Bloembergen, J. Ducuing and P. S. Pershan, "Interactions between light waves in a nonlinear dielectric," Phys. Rev. 127, 1918-1939 (1962).

7. O. Bang, "Dynamical equations for wave packets in material with both quadratic and cubic response," J. Opt. Soc. Am. B 14, 51-61 (1997).

8. H. He, P. D. Drummond and B. A. Malomed, "Modulational stability in dispersive optical systems with cascaded nonlinearity," Opt. Commun, 123, 394-402 (1996); H. He, A. Arraf, C. M. de Sterke, P. D. Drummond and B. A. Malomed, "Theory of Modulational instability in Bragg gratings with quadratic nonlinearity," Phys. Rev. E 59, 6064-6078 (1996). 